\title{
Agomelatine: The evidence for its place in the treatment of depression
}

This article was published in the following Dove Press journal:

Core Evidence

5 August 2009

Number of times this article has been viewed

\author{
Daniela Eser \\ Thomas C Baghai \\ Hans-Jürgen Möller \\ Department of Psychiatry \\ and Psychotherapy, \\ Ludwig-Maximilians-University, \\ Munich, Germany
}

Correspondence: Daniela Eser

Department of Psychiatry and

Psychotherapy, Ludwig-Maximilian-

University of Munich, Nußbaumstraße 7,

D-80336, Munich, Germany

Email Daniela.Eser@med.uni-muenchen.de
Introduction: Depressive disorders are among the main causes of disability due to disease. In spite of recent progress in the pharmacotherapy of depression, there is still a high nonresponse rate of $\sim 30 \%$ to the first antidepressant treatment. Furthermore, the latency of several weeks until sufficient clinical improvement and the risk of side effects remain unresolved problems. Therefore, there is still further need for the development of new antidepressants. In the last years a variety of melatonin receptor agonists have been synthesized and evaluated for the treatment of sleep disorders. Animal studies suggested that agomelatine (S-20098), a synthetic melatonergic $\mathrm{MT}_{1}$ and $\mathrm{MT}_{2}$ receptor agonist with serotonin receptor antagonistic properties, may have additional activating properties and may represent a new approach in the treatment of depression.

Aims: Clinical trials that have demonstrated efficacy and safety of agomelatine for the treatment of depression are reviewed.

Evidence review: In clinical trials, including phase III studies, superior efficacy compared to placebo and good efficacy compared to standard antidepressants was shown for agomelatine for the acute treatment of major depression. In all studies published so far agomelatine was safe and the overall tolerability profile was superior to selective serotonin reuptake inhibitors or selective serotonin and norepinephrine reuptake inhibitors.

Place in therapy: Agomelatine may represent a novel perspective in the treatment of acute depression. The improvement of sleep disturbances, the tolerability in terms of sexual side effects, and the lack of withdrawal symptoms after abrupt discontinuation of treatment may represent important clinical benefits compared to established antidepressants.

Keywords: agomelatine, antidepressants, melatonin, major depression, evidence

Core evidence place in therapy summary for agomelatine in major depression

\begin{tabular}{|c|c|c|}
\hline Outcome measure & Evidence & Implications \\
\hline \multicolumn{3}{|l|}{ Patient-oriented evidence } \\
\hline $\begin{array}{l}\text { Reduction of depressive } \\
\text { symptoms }\end{array}$ & Clear & $\begin{array}{l}\text { Agomelatine more effective than placebo and as effective } \\
\text { as paroxetine or venlafaxine }\end{array}$ \\
\hline Improvement of sleep & Clear & $\begin{array}{l}\text { Earlier and better improvement on subjective getting to sleep } \\
\text { and on subjective quality of sleep compared to venlafaxine }\end{array}$ \\
\hline \multicolumn{3}{|l|}{ Disease-oriented evidence } \\
\hline $\begin{array}{l}\text { Symptom relief in terms of } \\
\text { HAM-D reduction }\end{array}$ & Clear & $\begin{array}{l}\text { Agomelatine more effective than placebo and as effective } \\
\text { as paroxetine or venlafaxine }\end{array}$ \\
\hline Response to treatment & Clear & Agomelatine as effective as paroxetine and venlafaxine \\
\hline Remission after treatment & Clear & Agomelatine as effective as paroxetine and venlafaxine \\
\hline $\begin{array}{l}\text { Economic evidence } \\
\text { Cost effectiveness }\end{array}$ & $\begin{array}{l}\text { No } \\
\text { evidence }\end{array}$ & $\begin{array}{l}\text { So far, no information about costs of the drug on European } \\
\text { market. Studies required to validate assumption that } \\
\text { better tolerability profile will translate into improved cost } \\
\text { effectiveness }\end{array}$ \\
\hline
\end{tabular}

submit your manuscript | www.dovepress.com

Dovepress 


\section{Scope, aims, and objectives}

Melatonin secretion underlies strict circadian rhythms ${ }^{1}$ and is regulated via the cAMP signal transduction cascade. ${ }^{2}$ Several studies have reported beneficial effects of melatonin for the treatment of sleep disorders. However, the therapeutic use of melatonin, which is offered as a supplement in the US, is restricted by its low bioavailability. Therefore, several melatonin agonists have been developed in recent years and examined in clinical trials for the treatment of insomnia (for a current review of melatonin receptor agonist development, see Turek and colleagues ${ }^{3}$ ). To date, agomelatine (S-20098; Valdoxan ${ }^{\circledR}$, Servier), a synthetic melatonergic $\mathrm{MT}_{1}$ and $\mathrm{MT}_{2}$ receptor agonist, ${ }^{4}$ is the best characterized melatonin receptor agonist in terms of preclinical studies. In contrast to melatonin, agomelatine also possesses serotonin $\left(5-\mathrm{HT}_{2 \mathrm{C}}\right)$ receptor antagonistic properties ${ }^{5}$ within the central nervous system (CNS). Several preclinical studies indicated that agomelatine has activating ${ }^{6}$ and antidepressant effects, which are comparable to the antidepressant efficacy of established antidepressants. Therefore, agomelatine may represent a new approach for the treatment of depression. The efficacy and tolerability of agomelatine in the acute treatment of depressive disorders has been evaluated in several controlled clinical trials. In the following article the evidence for agomelatine's antidepressant efficacy will be reviewed and its place in the acute treatment of depression will be discussed.

\section{Methods}

The medical literature was reviewed for appropriate articles relating to agomelatine on PubMed (http://www.ncbi.nlm. nih. gov) using the search terms "agomelatine OR S-20098." The search was updated on September 1, 2008. One hundred and sixteen articles were found, of which 11 were written in languages other than English and were therefore excluded. Within those 105 publications, 12 reports from clinical trials and 24 review articles have been identified. Out of the reports from clinical trials, 10 are included and also cited in this review (Table 1). Additionally, congress proceedings focussing on the effects of agomelatine in the treatment of depression were also included where appropriate.

So far no pharamacoeconomic studies of agomelatine have been published. The results of the included clinical trials are reviewed, focussing on clinical efficacy, safety, and tolerability of agomelatine in the treatment of major depression.

\section{Disease overview}

Depressive disorders are among the main causes of disability due to disease and the World Health Organization (WHO)
Table I Evidence base included in the review

\begin{tabular}{lll}
\hline Category & \multicolumn{2}{l}{ Number of records } \\
\cline { 2 - 3 } & Full papers & Abstracts \\
\hline Initial search & 116 & \\
Records excluded & 11 & 4 \\
Records included & 105 & \\
Total records included & 10 & 0 \\
Level I clinical evidence & & 1 \\
(systematic review, meta analysis) & 0 & \\
Level 2 clinical evidence (RCT) & 5 & \\
Level $\geq 3$ clinical evidence & & 3 \\
Trials other than RCT & 2 & \\
Case reports & & \\
Other outcome than depression & 3 & 0 \\
Economic evidence & &
\end{tabular}

For definitions of levels of evidence, see Core Evidence website (http://dovepress. com/core-evidence-journal).

Abbreviation: $\mathrm{RCT}$, randomized controlled trial.

estimates that they will be the second most important cause of disability by the year 2020. ${ }^{7}$ Chronic depressive episodes are common and are associated with greater illness burden and socioeconomic disadvantage. ${ }^{8}$ Throughout Europe, $23 \%$ of years of healthy life are lost and approximately one-third of all burden of disease is caused by neuropsychiatric diseases. ${ }^{9}$ The 1-year prevalence of depression in Europe is around $5 \% .{ }^{10}$ The life time prevalence of depression varies widely from $3 \%$ in Japan to $16.9 \%$ in the US, with most countries in the range between $8 \%-12 \% .{ }^{11}$ Despite intensive biologically oriented psychiatric research over the last decades, the etiology of depressive disorders is not yet fully understood, although a multifactorial genesis is supposed. Besides psychologic and social factors, biologic variables apparently play a major role that lead to a disturbed CNS homeostasis. The so-called catecholamine- and serotonin-deficiency hypothesis, ${ }^{12}$ which postulates a deficiency of monoamines (norepinephrine and serotonin) within the synaptic cleft, plays a major role in the understanding of the pathophysiology of depression.

\section{Current therapy options}

The treatment of depressive disorders consists of a complex multimodal therapy that is determined by the current state of the illness. The treatment of depression includes pharmacotherapy, psychotherapy, and sociotherapy. Whereas pharmacotherapy is not always mandatory for less severe forms of depression, severe depression usually requires pharmacotherapy or electroconvulsive therapy. In addition, a variety 
of other biologic interventions, such as sleep deprivation and bright light therapy, may be of use in certain patient subgroups. The discovery of tricyclic antidepressants (TCAs) was a milestone in the treatment of depression. However, in spite of the undoubted effectiveness of TCAs it soon became apparent that their anticholinergic and antihistaminergic side effects may cause problems. As a consequence, new antidepressants were developed with a more selective mode of action, which mainly aimed at avoiding these side effects. Currently, tri- and tetracyclic antidepressants with predominant serotonergic, noradrenergic, or mixed serotonergic/noradrenergic action are available. In addition, selective and reversible inhibitors of the monoamine oxidase $\mathrm{A}$, an irreversible monoamine oxidase $\mathrm{B}$ inhibitor, nonselective and irreversible inhibitors of the monoamine oxidase, selective serotonin reuptake inhibitors (SSRI), selective norepinephrine reuptake inhibitors, and antidepressants with a dual mode of action such as selective serotonin and norepinephrine reuptake inhibitors (SNRI), and noradrenergic and specific serotonergic antidepressants acting via blockade of alfa ${ }_{2}$ and 5- $\mathrm{HT}_{2}$ receptors, are all available. ${ }^{13-17}$ The most recently investigated mechanism of action is the agonism at melatonergic $\mathrm{MT}_{1}$ and $\mathrm{MT}_{2}$ receptors and selective antagonism at serotonergic $5-\mathrm{HT}_{2 \mathrm{C}}$ receptors represented by the antidepressant agomelatine, ${ }^{5}$ which is currently under review by authorities in Europe.

\section{Unmet needs}

Although newer antidepressants are better tolerated and cause fewer side effects, their specific side-effect profile has to be taken into account during the treatment of depression. In addition, the latency of several weeks until the onset of sufficient therapeutic effects remains a serious and clinically relevant problem. This principle holds true for each antidepressant and each class of antidepressant mechanisms. A further general problem in pharmacotherapy of depression is the possible nonresponse to the first antidepressant treatment. ${ }^{18-20}$ Approximately $30 \%$ of depressed patients do not show sufficient improvement after the first course of an adequate antidepressant treatment and a further $20 \%$ discontinue due to tolerability problems. ${ }^{21}$ Adequacy of treatment includes the use of a treatment with proven efficacy during a time interval of at least 4-6 weeks in a sufficient therapeutic dose range including reliable patient adherence to therapy. ${ }^{19,22,23}$ Half of patients who do not respond adequately to a first course also fail to respond to a second antidepressant treatment trial. If several antidepressant treatment trials have been inefficacious, even lower response rates after switching to another drug may be observed. ${ }^{24}$

\section{Clinical evidence for agomelatine in the treatment of major depression}

Melatonin secretion underlies strict circadian rhythms ${ }^{1}$ and is regulated via the cAMP signal transduction cascade. ${ }^{2}$ A variety of animal studies and clinical trials in depressed patients suggest that agomelatine, a synthetic melatonergic $\mathrm{MT}_{1}$ and $\mathrm{MT}_{2}$ receptor agonist ${ }^{4}$ with $5-\mathrm{HT}_{2 \mathrm{C}}$ receptor antagonistic properties ${ }^{5}$ within the CNS, has activating and antidepressant effects. ${ }^{6}$

In several animal models of depression agomelatine has been shown to possess antidepressant effects that were comparable to those of established antidepressants. In contrast to melatonin the beneficial effects of agomelatine did not depend on the time of administration, indicating that besides the chronobiotic, other properties may contribute to its antidepressant-like activity. ${ }^{25}$ Furthermore, agomelatine has been shown to have chronobiotic activity similar to melatonin in animal models of disturbed circadian rhythms..$^{26,27}$

\section{Clinical efficacy assessment}

Clinical trials focus on symptomatic relief measured by specific scales, such as the Hamilton Depression Score (Hamilton Depression Rating Scale, HAM-D), ${ }^{28}$ and define response and remission in relation to these scores. The traditional definition of response to antidepressant therapy includes a 50\% improvement in symptoms (usually 50\% reduction of the baseline score). Remission is defined as the absence of depressive symptoms and a full return to premorbid levels of functioning. In most randomized controlled trials an absolute rating scale threshold is defined as remission (eg, HAM-D score $\leq 7$ ). Goals of clinical management can be divided into acute, intermediate, and long-term goals. The ultimate goal of acute treatment is to achieve remission, meaning not only being asymptomatic (in the sense of not meeting the criteria for diagnosis of the disorder and having no or only minimal residual symptoms) but also showing improvement in psychosocial and occupational functioning. The intermediate goal is further stabilization and prevention of a relapse, elimination of subsyndromal symptoms, and restoration of the prior level of functioning. The long-term goal is full recovery, ${ }^{29}$ to prevent further episodes, maintain functioning, and ensure a satisfactory quality of life. ${ }^{30-32}$

\section{Clinical efficacy of agomelatine in phase II and III trials Symptomatic relief}

The clinical efficacy of agomelatine in the treatment of depressive disorders was first investigated in a multinational, 
randomized, double-blind, placebo-controlled trial. ${ }^{33}$ In this study three different doses of agomelatine were compared with an active comparator. Seven hundred and eleven patients aged between 18 and 65 years, who were suffering from major depressive disorder $(n=698)$ or bipolar II depression $(\mathrm{n}=13)$ according to the Diagnostic and Statistical Manual of Mental Disorders, Fourth Edition (DSM-IV), ${ }^{34}$ with a minimum severity score of 22 in the 17 item HAM-D (HAM-D17) ${ }^{35}$ were treated with agomelatine, paroxetine, or placebo. After an obligatory 1-week placebo run-in period, patients were randomized to receive agomelatine 1,5 , or $25 \mathrm{mg}$ once daily in the evening, paroxetine $20 \mathrm{mg}$ /day in the morning, or placebo during the 8-week study period. After exclusion of placebo responders during the run-in period (defined as HAM-D17 improvement $>20 \%$ ) the mean HAM-D17 score at baseline was 27.4 and the mean Montgomery-Asberg Depression Rating Scale (MADRS) ${ }^{36}$ score was 31.5 , indicating a moderately to severely depressed study population. One-third of the patients had a depressive episode of severe intensity, defined as HAM-D17 score $\geq 25$. The treatment groups showed no significant differences in either demographic or clinical variables.

The intent-to-treat analysis using the last observation carried forward method in patients who had received at least one post-randomization treatment and had a baseline and at least one postbaseline rating, showed a statistically significant superiority in the mean scores of all agomelatine-treated patients taken together in comparison to the placebo-treated group. Subsequent distinct analyses of the three agomelatine treatment groups, however, showed clear effectiveness only in the patient group that received $25 \mathrm{mg} /$ day, whereas agomelatine 1 and $5 \mathrm{mg} /$ day did not differ from placebo. The time to first response was significantly shorter in the agomelatine $25 \mathrm{mg}$ /day group in comparison to placebo. In contrast to paroxetine, which first showed significant advantages after 4 weeks, agomelatine $25 \mathrm{mg}$ /day was significantly superior to placebo already after 2 weeks of treatment.

The clinical efficacy of agomelatine in major depression has been confirmed in further randomized, double-blind, placebo-controlled studies. In the study published by Kennedy and Emsley, ${ }^{37} 212$ outpatients were treated with agomelatine $25 \mathrm{mg} /$ day, with the possibility of increasing the dose to $50 \mathrm{mg} /$ day in case of insufficient improvement after 2 weeks of treatment. ${ }^{38}$ After a 6-week treatment period, agomelatine was significantly more effective in comparison with placebo. Patients treated with agomelatine had a significantly lower HAM-D score $(P=0.0017)$ at endpoint $(14.1 \pm 7.7)$ compared with patients receiving placebo $(16.5 \pm 7.4)$.
Furthermore, there was a significant improvement of severity of disease measured with the Clinical Global Impression Scale (CGI-S) score with agomelatine compared with placebo $(3.2 \pm 1.3$ versus $3.6 \pm 1.3 ; \mathrm{P}=0.0017)$. Thirty-six $(34 \%)$ patients in the agomelatine and $38(37 \%)$ patients in the placebo group failed to improve after 2 weeks of treatment. Therefore, in the agomelatine group the dosage was increased to $50 \mathrm{mg} / \mathrm{day}$, while placebo-treated patients continued to receive placebo. Also in this subgroup of increased agomelatine-treated patients, significant improvements in HAM-D score were observed at study endpoint. The HAM-D score decreased in the agomelatine-treated patients from $26.1 \pm 2.6$ at baseline to $17.5 \pm 7.4$ at week 6 , compared with a decrease from $26.7 \pm 2.8$ to $20.4 \pm 6.0$ in the placebo group.

In a further double-blind, parallel-group, flexible-dosing study, ${ }^{39}$ an interactive voice response system was used as a novel blinding method. Two hundred and thirty-eight patients with moderate-to-severe major depression were treated with agomelatine over 6 weeks. In case of insufficient improvement at week 2, dose adjustment from agomelatine 25 to $50 \mathrm{mg} /$ day was possible. In terms of HAM-D final scores agomelatine was significantly more efficacious than placebo with an agomelatine-placebo difference of $3.44(P=0.001)$. Furthermore, in terms of severity of disease measured with CGI-S, agomelatine significantly improved CGI and decreased CGI-severity compared with placebo.

Further evidence for the efficacy of agomelatine came from a double-blind, randomized study with the main goal of investigating the effects of agomelatine on sleep improvement. ${ }^{40}$ In this flexible-dose study, 332 outpatients suffering from major depression were treated with agomelatine $25-50 \mathrm{mg} /$ day or venlafaxine $75-150 \mathrm{mg} /$ day. After 6 weeks of treatment the antidepressant efficacy of agomelatine in terms of CGI global improvement was comparable to that of venlafaxine.

\section{Response and remission}

Analysis of response and remission rates during agomelatine treatment suggested that agomelatine exerts high efficacy in the treatment of depressive disorders. In the first randomized, double-blind, placebo-controlled trial of agomelatine ${ }^{33}$ response, defined by an improvement of 50\% in the HAM-D score, was achieved significantly more often in the agomelatine $25 \mathrm{mg} /$ day (61.5\%) and agomelatine $1 \mathrm{mg} /$ day (62.5\%) group in comparison with placebo $(46.3 \%)$ as shown by pairwise comparison. In contrast, neither agomelatine $5 \mathrm{mg} /$ day $(51.4 \%)$ nor paroxetine $(56.3 \%)$ was significantly superior to placebo. In this study remission defined by a HAM-D17 
score at endpoint below 7 was achieved significantly more often with agomelatine $25 \mathrm{mg} /$ day (30.4\%) and paroxetine $(25.7 \%)$ than with placebo (15.4\%).

Significant efficacy of agomelatine was also found in a randomized, double-blind, multicenter study of agomelatine versus paroxetine published with the main goal of investigating discontinuation symptoms in sustained remitted patients. ${ }^{41}$ During the first part of the study 335 outpatients with major depression (DSM-IV) with a mean MADRS score of 23 were treated after a drug-free run-in period with either agomelatine $25 \mathrm{mg} /$ day or paroxetine $20 \mathrm{mg}$ /day once daily for 12 weeks. One hundred and ninety-two patients achieved sustained remission, defined as an MADRS score $\leq 12$ at weeks 8,10 , and 12 . The rate of sustained remitters was $52.7 \%$ in the agomelatine and $61.9 \%$ in the paroxetine group. At the time of the second randomization, at entry into the discontinuation period, there were no statistically significant differences between the two groups.

Furthermore, in a recently published study by Kennedy et al, ${ }^{42}$ investigating sexual dysfunction in 276 depressed patients during antidepressant therapy with agomelatine $50 \mathrm{mg} /$ day or the SNRI venlafaxine extended release $150 \mathrm{mg} /$ day over a period of 12 weeks, comparable high remission rates (defined as an MADRS score $\leq 12$ ) of $73 \%$ in the agomelatine and $66.9 \%$ in the venlafaxine group have been observed.

Although it has to be considered that unequal definitions of remission, different dosages, and different treatment intervals might account for differences in the observed remission rates, these study results indicate that agomelatine exerts antidepressant efficacy that leads not only to treatment response but also to a rate of sustained remission similar to that of established antidepressants.

Response rates were also significantly higher with agomelatine $25-50 \mathrm{mg}$ /day (54.3\% and $49.1 \%$ ) than with placebo (35.5\% and 34.2\%) in two randomized, double-blind, flexible-dosing studies. ${ }^{37,39}$

\section{Specific subgroups of depressed patients}

The antidepressant efficacy of agomelatine has also been proven in the subgroup of severely depressed patients, defined by the generally accepted criterion of a baseline HAM-D17 score $\geq 25$.

In the study by Loo et $\mathrm{al}^{33}$ an analysis of the subgroup of severely depressed patients $(n=586)$ revealed that patients treated with agomelatine $25 \mathrm{mg}$ /day $(\mathrm{n}=120)$ but not those receiving the lower doses differed significantly from placebo-treated patients $(n=114)$ in terms of HAM-D final scores $(13.14 \pm 8.4$ versus $16.1 \pm 9.1)$. In contrast, the active comparator paroxetine was not significantly superior to placebo in this study population (HAM-D final score $14.1 \pm 8.4)$.

Similarly, in the study published by Kennedy and Emsley, ${ }^{37} 76$ severely depressed patients were treated with agomelatine and 75 severely depressed patients were treated with placebo. Treatment with agomelatine resulted in a significantly lower HAM-D score at endpoint compared with placebo $(14.4 \pm 7.9$ versus $17.3 \pm 7.2 ; P=0.024)$. Moreover, in the study population with severe depression, the difference in mean HAM-D score at endpoint between agomelatine and placebo exceeded the difference in the full study population $(2.72 \pm 1.19$ versus $2.30 \pm 1.02)$ indicating high efficacy of agomelatine in severely depressed patients.

In addition, first results from open-label studies indicated that agomelatine may not only be effective in the treatment of major depression, but also in the treatment of bipolar and seasonal affective disorder (SAD).

Agomelatine $25 \mathrm{mg}$ /day as an adjunctive therapy to lithium $(n=14)$ or valpromide $(n=7)$ significantly improved depressive symptoms in bipolar I patients after 6 weeks of treatment, ${ }^{43}$ and agomelatine $25 \mathrm{mg}$ /day yielded a significant decrease in depressive symptoms measured with the Structured Interview Guide for the HAM-D SAD-version, CGI-S, and CGI-I in 37 patients suffering from SAD. ${ }^{44}$

\section{Depression-associated sleep disturbances}

In view of its pharmacologic profile agomelatine is likely to possess sleep-wake cycle regulating properties. In several animal models of disturbed circadian rhythms agomelatine has been shown to have chronobiotic activity similar to melatonin. These results are also of particular interest for the treatment of affective disorders since a disorganization of circadian rhythms is suggested to play an important role in the pathophysiology of major depression. ${ }^{45,46}$

The influence of agomelatine $50 \mathrm{mg}$ /day on circadian rhythms was investigated in a double-blind, placebo-controlled, crossover study in healthy, older, adult men. ${ }^{47}$ At the end of the 15-day active treatment period, significant 2-hour phase advances in the 24-hour profiles of body temperature and cortisol levels were found. Similar trends were seen in thyroid stimulating hormone secretion. Polysomnographic investigations, however, showed no effects of agomelatine on normal sleep patterns suggesting that agomelatine facilitates the realignment of overt circadian rhythms in older adults.

In addition, in patients with major depression treated with agomelatine $25 \mathrm{mg}$, polysomnography shows regulation of 
sleep-wake rhythms increasing the duration of slow wave sleep and normalizing its distribution through the night. ${ }^{48}$ After 6 weeks of treatment sleep efficiency, time awake after sleep onset, and the total amount of slow wave sleep increased. In contrast agomelatine did not change rapid eye movement (REM) latency, amount of REM, or REM density. ${ }^{49}$

In addition, in a dose-finding study, ${ }^{33,50}$ somatic complaints and symptoms related to sleep disturbances decreased substantially throughout the study, further indicating that agomelatine has beneficial effects in the treatment of depression-related sleep disturbances.

In a double-blind, randomized study in 332 outpatients suffering from major depression, the effects of agomelatine 25-50 mg/day and venlafaxine 75-150 mg/day on subjective sleep quality were compared. In contrast to venlafaxine, agomelatine showed an earlier and better improvement on subjective getting to sleep and on subjective quality of sleep as well as a better improvement of daytime sleepiness. ${ }^{40}$ These results indicate that agomelatine may contribute to a normalization of disrupted circadian rhythms in depression via phase-shifting properties without direct sedation and therefore not disturbing the normal day-sleep rhythm.

\section{Safety and tolerability}

In all clinical trials published to date, agomelatine has a better safety and tolerability profile than established antidepressants, including the investigated SSRIs and SNRIs.

In a first dose-finding pilot study in 28 patients no modifications of cardiovascular parameters or biologic abnormalities were seen after a 4-8 week treatment period with agomelatine 5 or $100 \mathrm{mg} /$ day. ${ }^{33,50}$ Slightly more adverse events and severe treatment-related adverse events were seen in the $100 \mathrm{mg} /$ day group.

In the above mentioned large, double-blind study by the same authors, ${ }^{33} 56.5 \%$ of 711 patients reported at least one adverse event that emerged or worsened during the study such as headache, abdominal pain, nausea, diarrhea, somnolence, or anxiety. No significant difference between agomelatine and placebo was seen, whereas during paroxetine treatment significantly more gastrointestinal adverse events, predominantly nausea, were seen than with placebo. Again no clinically relevant changes in vital signs, weight, or cardiovascular parameters, including electrocardiogram recordings, were seen with agomelatine. In a further placebo-controlled trial, adverse events more common in the agomelatine group compared with placebo were dizziness (9.3\% versus $4.8 \%)$, nasopharingitis $(6.5 \%$ versus $3.8 \%)$, and influenza $(6.5 \%$ versus $2.9 \%) .{ }^{37}$
Remarkably, although agomelatine ameliorates sleep-related complaints, no sedation has been reported during the daytime. Impairment of sexual function has been reported for antidepressants and represents an important cause of noncompliance. ${ }^{51}$ Therefore, the effects of agomelatine on sexual function were studied in a specific trial. ${ }^{42,52}$ Using a sexual function questionnaire, sexual dysfunction was compared between agomelatine and venlafaxine extended release. ${ }^{42}$ Treatment-emergent sexual dysfunction was significantly less prevalent in the agomelatine group. Desire, arousal, and orgasm scores and the total sexual dysfunction score was significantly greater in venlafaxine-treated patients. In order to distinguish between medication-induced sexual side effects and sexual symptoms associated with depression an additional analysis of sexually active patients who achieved remission (defined by a decrease of at least $50 \%$ in the MADRS score compared to baseline and a total MADRS score $\leq 12$ after 10 weeks of treatment) was carried out. In this analysis $7.3 \%$ of agomelatine-treated patients compared with $15.7 \%$ of venlafaxine recipients reported a deterioration of sexual function. While men more often reported a decline in drive and desire, women more often reported deterioration of orgasm.

Discontinuation symptoms were investigated in a randomized, placebo-controlled, double-blind study ${ }^{41}$ in 88 patients with major depression who were sustained responders after agomelatine $25 \mathrm{mg}$ /day treatment lasting 12 weeks (the control group received paroxetine $20 \mathrm{mg}$ /day). Sixty-one patients continued agomelatine whereas 27 were abruptly switched to placebo. After 1 week of treatment interruption no statistically significant difference in the number of discontinuation symptoms between patients treated with agomelatine and those receiving placebo was observed. In contrast to these findings, 43 patients discontinuing the SSRI paroxetine experienced significantly more discontinuation symptoms, such as insomnia, muscle aches, dizziness, and nausea, than another 61 patients who continued treatment with the SSRI. After 2 weeks of treatment interruption the agomelatine group continued to show similar results to the placebo group and there were no significant differences between the paroxetine and the placebo group. No significant differences in the rates of relapse or partial relapse were registered in either of the active treatment groups or in the placebo group during the observation period of 2 weeks, which was relatively short for the estimation of the relapse risk. Nevertheless, patients who discontinued from paroxetine experienced a significantly greater worsening in CGI and MADRS scores in comparison to patients continuing 
the medication, which might be attributed to the emergent withdrawal symptoms in the paroxetine discontinuation group. Patients discontinuing agomelatine showed the same low severity of depressive symptoms as patients continuing the medication. During the discontinuation period no serious adverse events were recorded.

This study showed agomelatine was not associated with discontinuation symptoms during the observation period of 2 weeks. Because the half-life of agomelatine is 2 hours and the drug has no active metabolites, any discontinuation symptoms would be expected during the first week after discontinuation of the active drug. Therefore, the 2-week observation period seems to be long enough to rule out any emerging withdrawal or discontinuation symptoms. The inclusion of only sustained remitters rules out the likelihood of registering reemergent depressive symptoms during relapses or partial relapses as discontinuation symptoms. Therefore, agomelatine seems to be at lower risk for discontinuation symptoms after abrupt tapering, for example in noncompliant patients. This could be an important safety benefit during antidepressant therapies.

\section{Patient group/population}

Agomelatine has been shown to be effective in the treatment of major depression, especially in the subgroup of severely depressed patients. In a pooled analysis of placebo-controlled studies, agomelatine $25-50 \mathrm{mg}$ was significantly more effective than placebo. ${ }^{53}$ Agomelatine also ameliorates sleep-related complaints without sedation during the daytime suggesting that it may be of special benefit in agitated patients.

No large-scale studies on the long-term effects of agomelatine in the treatment of major depression have been published so no definite conclusion can be drawn at the moment in relation to long-term safety issues or efficacy in relapse prevention. Because of this, in 2006 the Committee for Medicinal Products for Human Use adopted a negative opinion, recommending the refusal of a marketing authorisation for the medicinal product Valdoxan/Thymanax ${ }^{\circledR}$ (Servier) for the treatment of depression.

However, in a double-blind multicenter study in depressed patients the efficacy of agomelatine in relapse prevention has been investigated. ${ }^{54}$ After initial response to agomelatine, 492 outpatients continued treatment with agomelatine or placebo. During the 6-month study period, $46.6 \%$ of patients receiving placebo, but only $21.7 \%$ of patients receiving agomelatine, relapsed. Therefore, these results indicate that agomelatine might also be effective in the long-term treatment of depression.

\section{Place in therapy}

Agomelatine is the first melatonergic and 5- $\mathrm{HT}_{2 \mathrm{C}}$ receptor antagonistic antidepressant. In all clinical trials published to date, including large-scale phase III studies, agomelatine has shown at least equal efficacy to active comparators such as SSRIs and SNRIs. Furthermore, agomelatine is also effective in the treatment of severe depression. In addition, agomelatine is the first antidepressant without antihistaminergic side effects that effectively ameliorates sleep-related complaints without sedation during the daytime.

In all clinical studies the overall tolerability profile of agomelatine was excellent and showed no impact on laboratory parameters, vital signs, blood pressure, heart rate, and QTc time intervals. The rate of treatment-emergent adverse events was not higher than with placebo. The sexual acceptability of agomelatine represents an important clinical benefit as well as the lack of withdrawal symptoms after abrupt discontinuation of treatment.

In summary, agomelatine represents a promising novel strategy in the treatment of depression. For clinicians it is a novel perspective in the acute treatment of depression that will hopefully show good clinical effectiveness paired with excellent tolerability also in the long-term treatment of depressed patients.

\section{Acknowledgments}

Daniela Eser has no potential conflict of interest. Thomas C. Baghai has accepted paid speaking engagements and acted as a consultant for AstraZeneca, Janssen-Cilag, Organon, Pfizer, and Servier. Hans-Jürgen Möller has received grant/research support, consulting fees, and honoraria from AstraZeneca, Bristol-Myers Squibb, Eli Lilly, GlaxoSmithKline, JanssenCilag, Lundbeck, MSD, Novartis, Organon, Otsuka, Pfizer, Schwabe, Sepracor, Servier, and Wyeth.

\section{References}

1. Lesieur D, Leclerc V, Chavatte P, Marcot C, Renard P, GuardiolaLemaitre B. Melatonin: a pertinent prototype for therapeutic intervention. Therapie. 1998;53:429-437.

2. Foulkes NS, Borjigin J, Snyder SH, Sassone-Corsi P. Rhythmic transcription: the molecular basis of circadian melatonin synthesis. Trends Neurosci. 1997;20:487-492.

3. Turek FW, Gillette MU. Melatonin, sleep, and circadian rhythms: rationale for development of specific melatonin agonists. Sleep Med 2004;5:523-532.

4. Zlotos DP. Recent advances in melatonin receptor ligands. Arch Pharm (Weinheim). 2005;338:229-247.

5. Millan MJ, Gobert A, Lejeune F, et al. The novel melatonin agonist agomelatine (S20098) is an antagonist at 5-hydroxytryptamine2C receptors, blockade of which enhances the activity of frontocortical dopaminergic and adrenergic pathways. J Pharmacol Exp Ther. 2003 306:954-964. 
6. Tuma S, Strubbe JJ, Mocaer E, Koolhaas JM. S20098 affects the free-running rhythms of body temperature and activity and decreases light-induced phase delay of circadian rhythms of the rat. Chronobiol Int. 2002;18:781-799.

7. WHO (World Health Organization). The Global Burden of Disease Project. 2002; Internet Communication.

8. Gilmer WS, Trivedi MH, Rush AJ, et al. Factors associated with chronic depressive episodes: a preliminary report from the STAR-D project. Acta Psychiatr Scand. 2005;112:425-433.

9. Olesen J, Leonardi M. The burden of brain diseases in Europe. Eur $J$ Neurol. 2003;10:471-477.

10. Paykel ES, Brugha T, Fryers T. Size and burden of depressive disorders in Europe. Eur Neuropsychopharmacol. 2005;15:411-423.

11. Andrade L, Caraveo-Anduaga JJ, Berglund P, et al. The epidemiology of major depressive episodes: results from the International Consortium of Psychiatric Epidemiology (ICPE) Surveys. Int J Methods Psychiatr Res. 2003;12:3-21.

12. Burke MJ, Preskorn SH. Short term treatment of mood disorders with standard antidepressants. In FE Bloom, DJ Kupfer, editors. Psychopharmacology: the fourth generation of progress. (p. 1053-1065). New York: Raven Press; 1995. p. 1053-1065.

13. Stahl SM. Selecting an antidepressant by using mechanism of action to enhance efficacy and avoid side effects. J Clin Psychiatry. 1998;59:23-29.

14. Kent JM. SNaRIs, NaSSAs, and NaRIs: new agents for the treatment of depression. Lancet. 2000;355:911-918.

15. Blakely RD. Physiological genomics of antidepressant targets: keeping the periphery in mind. $J$ Neurosci. 2001;21:8319-8323.

16. Frazer, A. Serotonergic and noradrenergic reuptake inhibitors: prediction of clinical effects from in vitro potencies. J Clin Psychiatry. 2001;62:16-23.

17. Pacher P, Kohegyi E, Kecskemeti V, Furst S. Current trends in the development of new antidepressants. Curr Med Chem. 2001;8:89-100.

18. Nierenberg AA, Amsterdam JD. Treatment-resistant depression: definition and treatment approaches. $J$ Clin Psychiatry. 1990;51 (Suppl):39-47.

19. Sackeim HA. The definition and meaning of treatment-resistant depression. J Clin Psychiatry. 2001;62(Suppl 16):10-17.

20. Charney DS, Grothe DR, Smith SL, et al. Overview of psychiatric disorders and the role of newer antidepressants. J Clin Psychiatry. 2002;63:3-9.

21. Sartorius N, Baghai TC, Baldwin DS, et al. Antidepressant medications and other treatments of depressive disorders: a CINP Task Force report based on a review of evidence. Int J Neuropsychopharmacol. 2007;10: S1-S207.

22. Fava GA. Can long-term treatment with antidepressant drugs worsen the course of depression? J Clin Psychiatry. 2003;64:123-133.

23. Kupfer DJ, Charney DS. "Difficult-to-treat depression." Biol Psychiatry. 2003;53:633-634.

24. Fava M, Rush AJ, Wisniewski SR, et al. A comparison of mirtazapine and nortriptyline following two consecutive failed medication treatments for depressed outpatients: a STAR*D report. Am J Psychiatry. 2006;163:1161-1172.

25. Bourin M, Mocaer E, Porsolt R. Antidepressant-like activity of S 20098 (agomelatine) in the forced swimming test in rodents: involvement of melatonin and serotonin receptors. J Psychiatry Neurosci. 2004;29: $126-133$.

26. Armstrong SM, McNulty OM, Guardiola-Lemaitre B, Redman JR. Successful use of S20098 and melatonin in an animal model of delayed sleep-phase syndrome (DSPS). Pharmacol Biochem Behav. 1993;46:45-49.

27. Redman JR, Guardiola-Lemaitre B, Brown M, Delagrange P, Armstrong SM. Dose dependent effects of S-20098, a melatonin agonist, on direction of re-entrainment of rat circadian activity rhythms. Psychopharmacology (Berl). 1995;118:385-390.

28. Hamilton M. Development of a rating scale for primary depressive illness. Br J Soc Clin Psychol. 1967;6:278-296.
29. Rush AJ, Kraemer HC, Sackeim HA, et al. Report by the ACNP Task Force on response and remission in major depressive disorder. Neuropsychopharmacol. 2006;31:1841-1853.

30. AHCPR (Agency for Health Care Policy and Research). Evidence report on Treatment of depression: Newer Pharmacotherapies. 1999 Washington DC. Available at http://www.ahrq.gov/clinic/epcsums/ deprsumm.htm

31. APA (American Psychiatric Association). Practice guideline for the treatment of patients with major depressive disorder (revision). Am J Psychiatry. 2000a; 157:1-45.

32. Bauer M, Whybrow PC, Angst J, Versiani M, Möller H-J; WFSBP Task Force on Treatment Guidelines for Unipolar Depressive Disorders. World Federation of Societies of Biological Psychiatry (WFSBP) guidelines for biological treatment of unipolar depressive disorder, part 1: acute and continuation treatment of major depressive disorder. World J Biol Psychiatry. 2002;3:5-43.

33. Loo H, Hale A, D'haenen H. Determination of the dose of agomelatine, a melatoninergic agonist and selective 5-HT(2C) antagonist, in the treatment of major depressive disorder: a placebo-controlled dose range study. Int Clin Psychopharmacol. 2002;17:239-247.

34. APA (American Psychiatric Association). Diagnostic and Statistical Manual of Mental Disorders, Fourth Edition. Washington DC: American Psychiatric Association; 2000b.

35. Hamilton M. The Hamilton rating scale for depression. In: Sartorius N, Ban TA, editors. Assessment of Depression. Berlin; Springer: 1986. p. 143-152.

36. Montgomery SA, Asberg M. A new depression scale designed to be sensitive to change. BrJ Psychiatry. 1979;134:382-389.

37. Kennedy SH, Emsley R. Placebo-controlled trial of agomelatine in the treatment of major depressive disorder. Eur Neuropsychopharmacol. 2006;16:93-100.

38. Olie JP, Emsley R. Confirmed clinical efficacy of agomelatine $(25-50 \mathrm{mg})$ in major depression: two randomized, double-blind, placebo-controlled studies. Eur Neuropsychopharmacol. 2005;15Abstract S416.

39. Olie JP, Kasper S. Efficacy of agomelatine, a MT1/MT2 receptor agonist with 5-HT ${ }_{2 \mathrm{C}}$ antagonistic properties, in major depressive disorder. Int J Neuropsychopharmacol. 2007;10:661-673.

40. Lemoine R Guilleminault C, Alvarez E. Improvement in subjective sleep in major depressive disorder with a novel antidepressant, agomelatine: randomized, double-blind comparison with venlafaxine. J Clin Psychiatry. 2007;68:1723-1732.

41. Montgomery SA, Kennedy SH, Burrows GD, Lejoyeux M, Hindmarch I. Absence of discontinuation symptoms with agomelatine and occurrence of discontinuation symptoms with paroxetine: a randomized, double-blind, placebo-controlled discontinuation study. Int Clin Psychopharmacol. 2004;19:271-280.

42. Kennedy SH, Rizvi S, Fulton K, Rasmussen J. A double-blind comparison of sexual functioning, antidepressant efficacy, and tolerability between agomelatine and venlafaxine XR. J Clin Psychopharmacol. 2008;28:329-333.

43. Calabrese JR, Guelfi JD, Perdrizet-Chevallier C; Agomelatine Bipolar Study Group. Agomelatine adjunctive therapy for acute bipolar depression: preliminary open data. Bipolar Disord. 2007;6: 628-635.

44. Pjrek E, Winkler D, Konstantinidis A, Willeit M, Praschak-Rieder N, Kasper S. Agomelatine in the treatment of seasonal affective disorder. Psychopharmacology (Berl). 2006;190:575-579.

45. Wehr TA, Wirz-Justice A. Circadian rhythm mechanisms in affective illness and in antidepressant drug action. Pharmacopsychiatria. 1982;15:31-39.

46. Wirz-Justice A. Biological rhythm disturbances in mood disorders. Int Clin Psychopharmacol. 2006;21(Suppl 1):S11-S15.

47. Leproult R, Van OA, L'hermite-Baleriaux M, Van CE, Copinschi G. Phase-shifts of 24-h rhythms of hormonal release and body temperature following early evening administration of the melatonin agonist agomelatine in healthy older men. Clin Endocrinol (Oxf). 2005;63: 298-304. 
48. Quera-Salva MA, Vanier B, Chapotot F, et al. Effect of agomelatine on the sleep EEG in patients with major depressive disorder. Eur Neuropsychopharmacol. 2005;15(Suppl 3):435.

49. Quera-Salva MA, Vanier B, Laredo J, et al. Major depressive disorder, sleep EEG and agomelatine: an open-label study. Int J Neuropsychopharmacol. 2007;10:691-696.

50. Loo H, Dalery J, Macher JP, Payen A. Pilot study comparing in blind the therapeutic effect of two doses of agomelatine, melatonin- agonist and selective 5HT2c receptors antagonist, in the treatment of major depressive disorders. Encephale. 2003;29:165-171.

51. Rosen RC, Lane RM, Menza M. Effects of SSRIs on sexual function: a critical review. J Clin Psychopharmacol. 1999;19:67-85.
52. Kennedy SH. Sexual function in remitted depressed patients following agomelatine and venlafaxine XR treatment. Eur Neuropsychopharmacol. 2005;15: Abstract S440.

53. Montgomery SA, Kasper S. Severe depression and antidepressants: focus on a pooled analysis of placebo-controlled studies on agomelatine. Int Clin Psychopharmacol. 2007;22:283-291.

54. Goodwin G, Rouillon F, Emsley R. Long-term efficacy of agomelatine, a novel antidepressant, in the prevention of relapse in out-patients with major depressive disorder. Eur Neuropsychopharm. 2007;17(Suppl 4): Abstract S361.

\section{Core Evidence}

\section{Publish your work in this journal}

Core Evidence is an international, peer-reviewed open-access journal evaluating the evidence underlying the potential place in therapy of drugs throughout their development lifecycle from preclinical to postlaunch. The focus of each review is to evaluate the case for a new drug or class in outcome terms in specific indications and patient

Submit your manuscript here: http://www.dovepress.com/core-evidence-journal

\section{Dovepress}

groups. The manuscript management system is completely online and includes a very quick and fair peer-review system, which is all easy to use. Visit http://www.dovepress.com/testimonials.php to read real quotes from published authors. 